\title{
IN VIVO AND IN VITRO INTERFERON (IFN) STUDIES WITH THE PORCINE REPRODUCTIVE AND RESPIRATORY SYNDROME VIRUS (PRRSV)
}

\author{
W. Buddaert, K. Van Reeth, and M. Pensaert \\ Laboratory of Veterinary Virology \\ Faculty of Veterinary Medicine \\ University of Gent \\ Salisburylaan 133 \\ B-9820 Merelbeke, Belgium
}

\section{ABSTRACT}

Some of the interactions between the porcine reproductive and respiratory syndrome virus (PRRSV) and the porcine interferon- $\alpha$ (IFN- $\alpha$ ) system were studied. In a first experiment, it was shown that pretreatment of primary porcine alveolar macrophages (AMs) with recombinant porcine ( $\mathrm{rPo}$ ) IFN- $\alpha 1$ resulted in significant reductions of PRRSV yield and numbers of antigen expressing cells. In a second experiment, sensitivity of PRRSV to IFN- $\alpha$ was confirmed in vivo. In pigs inoculated with porcine respiratory coronavirus (PRCV) - a potent inducer of endogenous IFN- $\alpha$ in the lungs of pigs-followed 2 days later by PRRSV-lung PRRSV titers were 1.7 to $2.9 \log _{10} \mathrm{TCID}_{50}$ reduced compared to those in singly PRRSV inoculated pigs. It was concluded therefore that PRRSV has a fairly good sensitivity to the antiviral effects of IFN- $\alpha$. A third experiment documented that in vivo PRRSV infection generally does not affect PRCV-induced IFN- $\alpha$ production in the lungs of pigs. In addition, it was shown that the IFN-inducing capacity of PRRSV is at least 159 times lower than that of PRCV. This finding suggests that cells other than AMs may be responsible for IFN production in the lungs of pigs.

\section{INTRODUCTION}

Infection with the porcine reproductive and respiratory syndrome virus (PRRSV) most frequently occurs via the respiratory route and the lungs are an important virus target 
organ. PRRSV replicates in the lungs during at least $3-4$ weeks and titers up to $10^{5.5}-10^{5.9}$ $\mathrm{TCID}_{50} / \mathrm{g}$ lung tissue are produced during the first 2 weeks. Macrophages of the alveolar spaces and alveolar septa play a prominent role in the pathogenesis of PRRSV infection. These cells make up $80-94 \%$ of the infected lung cells (Duan et al, 1997), are a major reservoir for virus in the lungs, and are a potential source of persistent viraemia. Cells of the macrophage type are quite intimately related tho the interferon (IFN) system. Macrophages are highly sensitive to the antiviral actions of interferons and, in turn, they are major IFN-producing cells in some animal species (Saksela et al., 1984).

Several porcine respiratory viruses are capable of inducing substantial amounts of biologically active interferon- $\alpha$ (IFN- $\alpha$ ) in the lungs of pigs. One very potent IFN-inducer is the porcine respiratory coronavirus (PRCV), and IFN titers up to 4000-5000 biological units $/ \mathrm{ml}$ are found in bronchoalveolar lavage fluids of experimentally infected pigs (Van Reeth and Pensaert, 1995). In dual infections with the PRCV followed by either H1N1-influenza virus or Aujeszky's disease virus (ADV), replication of the second virus in the lungs was significantly suppressed (Van Reeth and Pensaert, 1994; Van Reeth and Pensaert, 1996). This viral interference is likely mediated by IFN- $\alpha$.

Until recently, little attention has been paid to the interactions between PRRSV and the porcine interferon- $\alpha$ system (Albina et al., 1997). In a first part of this study, we have investigated whether PRRSV is sensitive to the antiviral actions of IFN- $\alpha$ in vitro and in vivo. In the in vitro studies, primary porcine alveolar macrophages and recombinant porcine IFN- $\alpha 1$ (rPo IFN- $\alpha) 1$ (Lefèvre et al., 1990) were used. In the in vivo studies, PRCV was used as an endogenous inducer of natural IFN- $\alpha$ in the lungs of pigs. In a second part of this study, we examined if a prior PRRSV infection affects lung IFN production by PRCV.

\section{METHODS AND MATERIALS}

\subsection{Antiviral Effect of IFN- $\alpha$ against PRRSV Replication in Vitro}

Primary porcine alveolar macrophages (AMs) were obtained by post mortem lung lavage of 4 to 6-week-old conventional pigs that were negative for PRRSV antibodies. Freshly seeded AMs were pretreated for $18 \mathrm{~h}$ with recombinant porcine IFN- $\alpha 1$ (generous gift from C. La Bonnardière, INRA, Jouy-en-Josas, France) at 1000 units $/ 10^{6}$ AMs or left untreated (virus controls). The IFN-treated and control wells were then rinsed with RPMI and inoculated with the Lelystad virus strain of PRRSV or with vesicular stomatitis virus

Table 1a. Kinetics of PRRSV production in alveolar macrophages pretreated with rPo IFN- $\alpha$

\begin{tabular}{llccccc}
\hline & & \multicolumn{5}{c}{ Virus titer ${ }^{\mathrm{a}}\left(\log _{10} \mathrm{TCID}_{50} / \mathrm{ml}\right)$ at $\ldots \mathrm{h}$ PI } \\
\cline { 3 - 7 } & Treatment & 1 & 8 & 18 & 30 & 42 \\
\hline \multirow{2}{*}{ PRRSV } & Control & 3.2 & 3.2 & 4.5 & 5.0 & 5.6 \\
& IFN- $\alpha$ & 3.5 & 2.9 & 2.3 & 2.4 & 3.0 \\
\multirow{4}{*}{ VSV } & & & & & \\
& Control & 4.5 & 6.3 & 7.3 & 7.8 & 8.5 \\
& IFN- $\alpha$ & 3.8 & 3.9 & 3.6 & 4.2 & 4.8 \\
\hline a
\end{tabular}

${ }^{\mathrm{a}}$ Means of 2 experiments. 
Table 1b. Kinetics of PRRSV antigen expression in alveolar macrophages pretreated with rPo IFN- $\alpha$

\begin{tabular}{lccccccc}
\hline & & \multicolumn{6}{c}{ IPMA positive cells ${ }^{\mathrm{a}}$ at $\ldots \mathrm{h}$ PI } \\
\cline { 3 - 8 } & Treatment & 1 & 12 & 18 & 30 & 48 & 72 \\
\hline PRRSV & Control & 0 & $15-26$ & $56-173$ & $264-301$ & CPE $^{\mathrm{b}}$ & $\mathrm{CPE}$ \\
& IFN- $\alpha$ & 0 & 0 & $0-4$ & $1-5$ & $146-212$ & $\mathrm{CPE}$ \\
\hline
\end{tabular}

(VSV) (MOI 0.04). VSV was included because of its known high sensitivity to porcine IFN- $\alpha$. After $2 \mathrm{~h}$ adsorption, the virus inoculum was removed and maintenance medium added. At different times post inoculation (PI), IFN-treated and control AM cultures were frozen $\left(-70^{\circ} \mathrm{C}\right)$ and thawed $(2$ cycles $)$ and infectious virus titers determined by titration in AM (PRRSV) and swine testicle (ST) (VSV) cell cultures.

In a parallel series of experiments, PRRS viral antigen expression in IFN-pretreated and control AM cultures was quantitated by an immunoperoxydase monolayer assay (IPMA) (Wensvoort et al., 1991).

\subsection{Antiviral Effect of Interferon against PRRSV Replication in the Lungs of Pigs}

Conventional 10-week-old pigs from herds free of PRRSV and PRCV were used. Two pigs were inoculated with PRCV (91V44 strain; $10^{7} \mathrm{TCID}_{50} / \mathrm{pig}$ ) followed 2 days later by PRRSV (Lelystad virus strain; $10^{5.0} \mathrm{TCID}_{50} / \mathrm{pig}$ ) and euthanatized 2 and 3 days PI (DPI) with PRRSV respectively (Table 2). Two singly PRCV inoculated pigs and 2 singly PRRSV inoculated pigs were euthanatized at corresponding times PI. Two uninoculated control pigs were included. PRRSV and PRCV titers in the left apical and diaphragmatic lung lobes were determined by titrations in AMs and ST cells respectively. The right lung

Table 2. Virus titers and IFN- $\alpha$ titers in the lungs of dually PRCV-PRRSV and singly virus-inoculated pigs

\begin{tabular}{|c|c|c|c|c|c|c|}
\hline \multirow[b]{3}{*}{ Inoculation } & \multirow{2}{*}{\multicolumn{2}{|c|}{$\begin{array}{l}\text { Euthanasia } \\
(\text { DPI })^{\mathrm{a}}\end{array}$}} & \multicolumn{3}{|c|}{ Virus titer $^{b}$} & \multirow{3}{*}{$\begin{array}{l}\text { IFN- } \alpha \text { titer } \\
\text { (U/ml BAL } \\
\text { fluid) }\end{array}$} \\
\hline & & & \multirow{2}{*}{$\frac{\text { PRCV }}{\text { Apic }^{c}}$} & \multicolumn{2}{|c|}{ PRRSV } & \\
\hline & PRCV & PRRSV & & Apic & Diaphr ${ }^{d}$ & \\
\hline \multirow[t]{2}{*}{ none } & $-^{\mathrm{e}}$ & - & neg & neg & neg & neg \\
\hline & - & - & neg & neg & neg & 111 \\
\hline \multirow[t]{2}{*}{ PRCV } & 4 & - & 6.7 & neg & neg & 406 \\
\hline & 5 & - & 7.2 & neg & neg & 455 \\
\hline \multirow[t]{2}{*}{ PRRSV } & - & 2 & neg & 5.5 & 5.2 & 219 \\
\hline & - & 3 & neg & 5.9 & 4.9 & 98 \\
\hline PRCV-2d- & 4 & 2 & 6.8 & 3.7 & 3.7 & 3281 \\
\hline PRRSV & 5 & 3 & 7.5 & 2.7 & 2.7 & 7014 \\
\hline
\end{tabular}

${ }^{\mathrm{a}}$ Time of euthanasia in days post inoculation.

${ }^{b}$ Virus titer in $\log _{10} \mathrm{TCID}_{50} / \mathrm{g}$ lung.

${ }^{c . d}$ Apical, diaphragmatic lung lobes.

${ }^{\mathrm{e}}$ Not applicable. 
was lavaged and bronchoalveolar lavage (BAL) fluid IFN titers were determined in a virus CPE inhibition assay with Madin-Darby bovine kidney (MDBK) cells and VSV.

\subsection{Effect of PRRSV Infection on the Ability of PRCV to Induce IFN- $\alpha$ in the Lungs}

Caesarian-derived colostrum-deprived (CDCD) pigs were used to avoid eventual IFN production by concurrent natural infections. Pigs were inoculated at the age of 3 weeks by the intratracheal route. Four pigs were dually inoculated with PRRSV first followed 3 ( 2 pigs) or 7 ( 2 pigs) days later by PRCV (Table 3 ). One pig of each group was euthanatized 3 and 4 DPI with PRCV. Singly PRCV (2 pigs) and PRRSV (2 pigs) inoculated control pigs were killed at corresponding times PI. Two inoculated control pigs were included. IFN titers in BAL fluids and virus titers were determined as described higher.

\section{RESULTS}

\subsection{In Vitro Sensitivity of PRRSV to IFN- $\alpha$}

Pretreatment of AMs with rPo IFN- $\alpha$ resulted in reduced PRRSV yields (Table 1a) and number of infected cells (Table $1 \mathrm{~b}$ ). Maximal reduction in virus yield was $2.6 \log _{10}$ $\mathrm{TCID}_{50}$ for PRRSV, compared to $3.7 \log _{10} \mathrm{TCID}_{50}$ for VSV. In IFN-treated AMs, titers of both viruses gradually increased between 18 and $42 \mathrm{~h}$ PI. Numbers of PRRSV infected cells in IFN-treated AM cultures were at least 53 times reduced at $30 \mathrm{~h}$ PI and increased thereafter. On the whole, IFN-treated AMs showed a $24 \mathrm{~h}$ delay in PRRSV antigen expression and CPE onset.

\subsection{In Vivo Sensitivity of PRRSV to IFN- $\alpha$}

Uninoculated control pigs were negative for PRCV and PRRSV. One of 2 pigs had an IFN- $\alpha$ titer of $111 \mathrm{U} / \mathrm{ml}$ BAL fluid.

While singly PRRSV inoculated pigs were negative for PRCV, PRCV titers in dually PRCV-PRRSV and singly PRCV inoculated pigs were similar (Table 2). Prior inoculation

Table 3. Interferon- $\alpha$ titers in BAL fluids of dually PRRSV-PRCV and singly virus-inoculated pigs

\begin{tabular}{|c|c|c|c|}
\hline \multirow[b]{2}{*}{ Inoculation } & \multicolumn{2}{|c|}{ Euthanasia (DPI) $^{\mathrm{a}}$} & \multirow{2}{*}{$\begin{array}{c}\text { IFN- } \alpha \text { titer } \\
\text { (U/ml BAL fluid) }\end{array}$} \\
\hline & PRRSV & PRCV & \\
\hline PRCV & $-b$ & 3 & 27171 \\
\hline PRCV & - & 4 & 9229 \\
\hline PRRSV & 7 & - & 58 \\
\hline PRRSV & 10 & - & 32 \\
\hline PRRSV-3d-PRCV & 6 & 3 & 53248 \\
\hline PRRSV-3d-PRCV & 7 & 4 & 649 \\
\hline PRRSV-7d-PRCV & 10 & 3 & 28535 \\
\hline PRRSV-7d-PRCV & 11 & 4 & 5114 \\
\hline
\end{tabular}

${ }^{a}$ Time of euthanasia in days post inoculation.

${ }^{\mathrm{b}}$ Not applicable. 
of pigs with PRCV resulted in mean reductions in PRRSV titer of 1.7 and $2.9 \log _{10} \mathrm{TCID}_{50}$ at 2 and 3 DPI with PRRSV respectively. IFN- $\alpha$ titers in the latter pigs were 15 and 72 times higher than those after inoculation with PRRSV only.

\subsection{Effect of PRRSV Infection on the Ability of PRCV to Induce IFN- $\alpha$ in the Lungs}

None of the uninoculated control pigs had any detectable IFN- $\alpha$ production.

All 3 groups of virus inoculated pigs were positive for IFN- $\alpha$ (Table 3 ). IFN- $\alpha$ titers reached up to $27171 \mathrm{U} / \mathrm{ml}$ BAL fluid in the singly PRCV inoculated pigs, but did not exceed $58 \mathrm{U} / \mathrm{ml}$ in the singly PRRSV inoculated pigs. As in singly PRCV inoculated pigs, IFN- $\alpha$ titers of dually PRRSV-3d-PRCV and PRRSV-7d-PRCV inoculated pigs were highest 3 DPI with PRCV. At that time, IFN- $\alpha$ titers amounted to 53248 and $28535 \mathrm{U} / \mathrm{ml}$ and were thus comparable to those detected after single PRCV inoculation.

\section{DISCUSSION}

The results presented here clearly demonstrate that PRRSV replication in AMs is sensitive to the antiviral effect of IFN- $\alpha$ in vitro and in vivo. The in vitro experimental conditions were selected on the basis of scientific and practical arguments. The natural virus host cell and species specific recombinant IFN- $\alpha$ were used. Interferon was used at a dose that can be achieved in the lungs of pigs by IFN induction or administration. While it could have been more appropriate to use an MOI of 1 to test the effect of IFN on one single cycle of virus replication, a relatively low MOI of 0.04 was used because of limitations in the PRRS virus stock titer. Furthermore, preliminary experiments had shown that IFN treatment before and after viral challenge was far more effective than pretreatment only. Such continuous treatment of AMs, however, hampered exact quantitations of infectious virus yield and viral antigen-expressing cells. Indeed, carry over of biologically active IFN from AMs in the antiviral assay into AM indicator cultures was demonstrated. Also, prolonged IFN treatment ( $>48 \mathrm{~h}$ ) caused a slight degree of toxicity in some AM cultures and thus interfered with interpretation of the IPMA staining. Consequently, IFN-pretreatment was selected for further experiments. All of these experimental conditions should be considered when making comparisons with previously determined sensitivities of other viruses. After all, compared to other antiviral studies with primary AMs and porcine (Esparza et al., 1988) or bovine viruses (Babiuk et al., 1985; Holland et al., 1991), PRRSV shows a fairly good sensitivity to IFN- $\alpha$. A "normal high sensitivity" of PRRSV to IFN- $\alpha$ was recently also reported by Albina and coworkers (1997). In vivo, using PRCV as an IFN inducer in the lungs of pigs, a maximal PRRSV yield reduction of $2.9 \log _{10}$ $\mathrm{TCID}_{50}$ could be obtained. Previously, similar experiments with H1N1-influenza virus (Van Reeth and Pensaert, 1994) and ADV (Van Reeth and Pensaert, 1996) had shown maximal yield reductions of 2.7 and $1.5 \log _{10} \mathrm{TCID}_{50}$ respectively. These in vivo differences between virus infections may depend largely upon their different sites of replication within the lungs.

Significant differences in IFN- $\alpha$ titers were found between the 2 in vivo experiments presented here. Maximal IFN titers detected were about $7000 \mathrm{U} / \mathrm{ml}$ in the first experiment and $53000 \mathrm{U} / \mathrm{ml}$ in the second experiment. In unpublished experiments with other viruses, we found that IFN titers following direct inoculation of virus into the trachea may be $\geq 10$ times higher than those after aerosol or intranasal virus inoculation. Most likely, the differ- 
ence in inoculation methods in the present experiments plays a major role in the observed differences in IFN production. Another difference between these 2 experiments is the sanitary status of the experimental pigs. In the PRCV-PRRSV dual infection experiment, we intended to mimic the field situation as closely as possible. Therefore, pigs originating from conventional breeding farms were used and these were transported to our isolation facilities 1 week before the start of the experiment. One of the control pigs, however, was positive for IFN- $\alpha$. Artursson and coworkers (1989) have demonstrated that $25 \%$ of $10-12$ week old conventional feeder pigs are positive for IFN- $\alpha$ in their serum within 5-10 days after regrouping in the fattening farm units. IFN production under these natural circumstances, as well as in our experiments, may result from virus infections or from stress caused by transport and adaptation to a new environment. Therefore, completely germfree caesarian derived and colostrum deprived pigs, reared in Horsefall-type isolation units, were used in our second experiment.

One of the most interesting aspects of this study was that even though PRRSV was a very poor IFN inducer in the lungs of pigs, it did not appear to have an inhibitory effect on IFN production by a subsequent PRCV infection. To our knowledge, the effect of viruses with AM tropism on lung IFN production by a second inducer has never been studied in vivo. In vitro, on the other hand, ADV (Iglesias et al., 1992) as well as African swine fever virus (Powell et al., 1996) infection of primary porcine AMs have been shown to interfere with IFN- $\alpha$ production by synthetic inducers. Even more important, transmissible gastrenteritis virus (TGEV)-induced IFN- $\alpha$ secretion by AMs was totally inhibited when cells were infected by PRRSV before (Albina et al., 1997). Our present in vivo findings can be explained in 2 ways. If IFN- $\alpha$ in the pig lung is produced by AMs, PRRSV clearly does not interfere with the IFN-producing capacity of its target cell in vivo. Another and more likely possibility is that lung cells other than AMs are responsible for IFN production. In pigs, so-called "natural IFN-producing cells" or null lymphocytes have been found to constitute a major subpopulation of IFN-producing leukocytes upon in vitro exposure to several viruses (Charley and Lavenant, 1990). Studies of the IFN- $\alpha$ producing cells in situ in the lungs of pigs are needed to clarify these issues.

\section{ACKNOWLEDGMENTS}

We thank Lieve Sys for skillful technical assistance. This study was financially supported by the Belgian Ministry of Agriculture.

\section{REFERENCES}

Albina, E., Carrat, C., and Charley, B., 1997, Interferon alpha response to porcine reproductive and respiratory syndrome virus (PRRSV), J. Gen. Virol., submitted.

Artursson, K., Wallgren, P., and Alm, G., 1989, Appearance of interferon- $\alpha$ in serum and signs of reduced immune function in pigs after transport and installation in a fattening farm, Vet. Immunol. Immunopathol. 23 : $345-353$.

Babiuk, L. A., Bielefeldt Ohmann, H., Gifford, G., Czarniecki, C. W., Scialli, V. T., and Hamilton, E. B., 1985, J. Gen. Virol. 66: 2383-2394.

Charley, B., and Lavenant, L.,1990, Characterization of blood mononuclear cells producing IFN- $\alpha$ following induction by coronavirus-infected cells (transmissible porcine gastroenteritis virus). Res. Immunol. 141: 141.

Duan, X., Nauwynck, H.J., and Pensaert, M.B.,1997, Virus quantification and identification of cellular targets in the lungs and lymphoid tissues of pigs at different time intervals after inoculation with porcine reproductive and respiratory syndrome virus (PRRSV), Vet. Microbiol. 56: 9-19. 
Esparza, I., Gonzalez, J. C., and Vinuela, E., 1988, Effect of interferon- $\alpha$, interferon- $\gamma$ and tumour necrosis factor$\alpha$ on African swine fever virus replication in porcine monocytes and macrophages, J. Gen. Virol. 69: 2973-2980.

Holland, S. P., Fulton, R. W., Short, E. C., Wyckoff, J. H., and Fox, J. C., 1991, In vitro and in vivo 2', 5'-oligoadenylate synthetase activity induced by recombinant DNA-derived bovine interferon $\alpha \mathrm{Il}$ in bovine alveolar macrophages and blood mononuclear cells, Am. J. Vet. Res. 52: 1779-1783.

Iglesias, G., Pijoan, C., and Molitor, T., 1992, Effects of pseudorabies virus infection upon cytotoxicity and antiviral activities of porcine alveolar macrophages, Comp. Immunol. Microbiol. Infect. Dis. 15: 249-259.

Lefèvre, F., L'Haridon, R., Borras-Cuesta, F., and La Bonnardière, C., 1990, Production, purification and biological properties of an Escherichia coli-derived recombinant porcine alpha interferon, J. Gen. Virol. 71: 1057-1063.

Powell, P.P., Dixon, L.K. and Parkhouse, R.M., 1996, An IkappaB homolog encoded by African swine fever virus provides a novel mechnanism for downregulation of proinflammatory cytokine responses in host macrophages, J. Virol. 70: 8527-8533.

Saksela, E., Virtanen, I., Hovi, T., Secher, D.S., and Cantell, K., 1984, Monocyte is the main producer of human alpha interferons following Sendai virus induction, Prog. Med. Virol. 30: 78.

Van Reeth, K., and Pensaert, M.B., 1994, Porcine respiratory coronavirus-mediated interference against influenza virus replication in the respiratory tract of feeder pigs, Am. J. Vet. Res. 55: 1275-1281.

Van Reeth, K., and Pensaert, M., 1995, Production of interferon- $\alpha$, tumor necrosis factor- $\alpha$ and interleukin-1 in the lungs of pigs infected with the porcine respiratory coronavirus. Proc. 3rd Congress Europ. Soc. Vet. Virol., Interlaken, Switzerland, 197-201.

Van Reeth, K., and Pensaert, M., 1996, A clinical and virological study in pigs infected with Aujeszky's disease virus shortly after infection with porcine respiratory coronavirus. Proc. 14th Congress Int. Pig Vet. Soc., Bologna, Italy, 137.

Wensvoort, G., Terpstra, C., Pol, J.M.A., ter Laak, E.A., Bloemraad, M., de Kluyver, E.P., Kragten, C., van Buiten, L., den Besten, A., Wagenaar, F., Broekhuijsen, J.M., Moonen, P.L.J.M., Zetstra, T., de Boer, E.A., Tibben, H.J., de Jong, M.F., van Veld, P., Groenland, G.J.R., van Gennep, J.A., Voets, M.T., Verheijden, J.H.M., and Braanskamp, J., 1991, Mystery swine disease in The Netherlands: the isolation of the Lelystad virus, Vet. Q. 13: $121-130$. 